\title{
Gait analysis methods in rehabilitation
}

\author{
Xiaotian Chang* \\ Professor, Shandong Academy of Medical Sciences Texas Tech \\ University, China
}

\section{Corresponding Author* \\ Xiaotian Chang, Professor, \\ Shandong Academy of Medical SciencesTexas Tech University, China}

Copyright: 2021 Xiaotian Chang. This is an open-access article distributed under the terms of the Creative Commons Attribution License, which permits unrestricted use, distribution, and reproduction in any medium, provided the original author and source are credited.

Received 01 Mar 2021; Accepted 16 Mar 2021; Published 22 Mar 2021

\section{Introduction}

Brand's four reasons for clinical tests and his analysis of the characteristics of valid biomechanical tests to be used in orthopaedics are taken as a basis for determinant what methodologies are needed for gait analysis during a clinical rehabilitation context.

\section{Measurement strategies in clinical gait analysis}

The state of the art of optical systems capable of measure the positions of retro-reflective markers placed on the skin is sufficiently advanced that they're most likely not a big supply of error in clinical gait analysis. Determinant the measurement of the topic and compensating for soft tissue movement in reference to the under-lying bones are currently the principal issues. Techniques for exploitation purposeful tests to see joint centres and axes of rotation are getting down to be used with success. Most likely the last nice challenge for optical systems is in exploitation process techniques to make amends for soft tissue measurements.
Within the long run future it's attainable that direct imaging of bones and joints in 3 dimensions (using imaging or fluoroscopy) could replace marker based mostly systems.

\section{Methods for decoding gait analysis knowledge}

There is still not associate in Nursing accepted general theory of why we tend to walk the means we tend to do. Within the absence of this, several explanations of walking address the mechanisms by that specific movements are achieved by explicit muscles. an entire new methodology is developing to see the functions of individual muscles. This desires additional development and validation. a selected demand is for subject specific models incorporating three-D imaging knowledge of the muscular-skeletal anatomy with kinematic and kinetic knowledge.

\section{Methods for understanding the results of intervention}

Clinical gait analysis is extraordinarily restricted if it doesn't permit clinicians to settle on between various attainable interventions or to predict outcomes. This will be achieved either by strictly planned clinical trials or exploitation theoretical models. The proof base is mostly poor partially attributable to the restricted range of prospective clinical trials that are completed and a lot of such studies ar essential. Terribly recent work has begun to show the potential of exploitation models of the mechanisms by which individuals with pathology get into order to simulate completely different potential interventions. The event of those models offers right smart promise for brand spanking new clinical applications of gait analysis. 